\title{
Design and fabrication of an integrated microsystem for microcapillary electrophoresis
}

\author{
Yick Chuen Chan ${ }^{1}$, Maria Carles ${ }^{2,3}$, Nikolaus J Sucher ${ }^{2,4}$, \\ Man Wong ${ }^{5}$ and Yitshak Zohar ${ }^{1}$ \\ ${ }^{1}$ Department of Mechanical Engineering, Hong Kong University of Science and Technology, \\ Clear Water Bay, Kowloon, Hong Kong Special Administrative Region \\ ${ }^{2}$ Biotechnology Research Institute, Hong Kong University of Science and Technology, \\ Clear Water Bay, Kowloon, Hong Kong Special Administrative Region \\ ${ }^{3}$ Department of Biochemistry, Hong Kong University of Science and Technology, \\ Clear Water Bay, Kowloon, Hong Kong Special Administrative Region \\ ${ }^{4}$ Department of Biology, Hong Kong University of Science and Technology, \\ Clear Water Bay, Kowloon, Hong Kong Special Administrative Region \\ ${ }^{5}$ Department of Electrical and Electronic Engineering, Hong Kong University of Science and \\ Technology, Clear Water Bay, Kowloon, Hong Kong Special Administrative Region
}

Received 12 March 2003, in final form 30 June 2003

Published 14 August 2003

Online at stacks.iop.org/JMM/13/914

\begin{abstract}
A capillary electrophoresis microsystem integrated with feed-through platinum electrodes was designed and fabricated for the separation of DNA fragments. A novel glass-to-silicon bonding technology, which allows anodic bonding of a glass wafer to a silicon wafer coated with a thick dielectric film by the inclusion of a thin intermediate amorphous silicon layer, was developed and employed to construct the microsystem. Despite the existence of a thick insulating material and non-uniform topography, robust devices without fluid leakage were obtained. Electrophoretic manipulation and separation of DNA fragments after capillary pre-treatment have been demonstrated and several operational considerations are discussed. The system performance suggests that silicon-based microsystems can be advantageous and practical for the fabrication of integrated microcapillary electrophoresis devices.
\end{abstract}

\section{Introduction}

Benefiting from the rapid progress in micromachining technology, the advancement and deployment of microfluidic systems in biotechnology applications have attracted major research and commercial interest. In recent years, a great deal of effort has been dedicated to the development of a miniaturized laboratory that integrates several biological assay tasks into a single device. The microcapillary electrophoresis (MCE) system, one of the major components of this lab-on-achip concept, has thus received particular attention.

Although genetic diagnosis using MCE has been performed for more than a decade, many systems available today are still relatively simple. Integrated fabrication technologies that accommodate fluid transport as well as thermal and detection functions on a common substrate are still lacking [1]. The majority of the early MCE devices have been fabricated using bonded and bulk micromachined glass substrates with external driving electrodes and detection systems [2-4]. Although higher voltages can be applied, external large-scale detection systems are required in those glass-based devices. More recently, the driving electrodes as well as electrochemical sensors for detection have been integrated on a single chip [5-7]. In spite of this, the major drawback of the incompatibility of the substrate with the conventional microelectronic fabrication technology still limits their potential of becoming truly lab-on-a-chip microsystems.

Silicon-based microfluidic systems are also designed and fabricated for MCE applications. The use of silicon 
Design and fabrication of an integrated microsystem for microcapillary electrophoresis

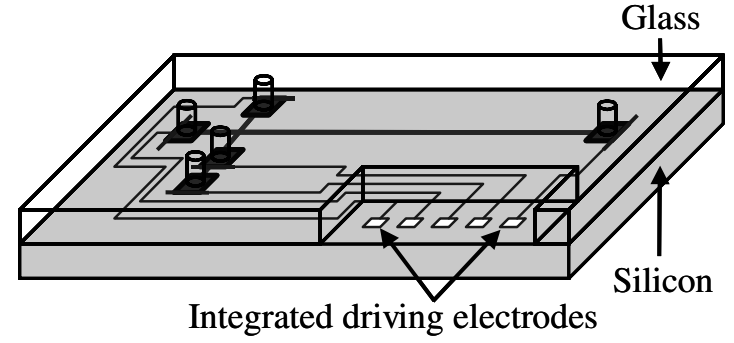

Figure 1. The conceptual design of an integrated MCE device.

instead of glass substrates allows the integration of on-chip electronic components and circuits. Many of those devices are constructed by bonding together a pair of silicon and glass substrates. In this approach, the relatively low-temperature anodic bonding technique is favourable, especially when metallization is involved. Different types of silicon-based MCE systems have been constructed in this approach [8-10]. Typically, an oxide layer is grown on the silicon substrate, prior to the bonding process, in order to insulate the semiconducting substrate from the running electrophoresis buffer. However, this oxide layer greatly reduces the yield and quality of the anodic bonding. It has been reported that an oxide layer exceeding $0.3 \mu \mathrm{m}$ in thickness could hardly achieve hermetic or strong anodic bonding [11]. We should keep in mind that an oxide layer a few thousand angstroms thick can only sustain a relatively low voltage before breakdown. Consequently, silicon-based microfluidic systems are considered to be of little practical use if the voltage breakdown problem cannot be resolved.

Aside from the voltage breakdown problem, silicon-based MCE systems do have the distinct advantage of CMOS process compatibility. Furthermore, the silicon substrate provides a path for higher heat dissipation rate when local heating becomes a severe obstacle. Recently, silicon-based MCE devices have been demonstrated with integrated DNA sensors utilizing surface micromachining techniques [12, 13]. The walls of the microcapillaries were constructed using a thin polymeric material with photoresist serving as the sacrificial layer. The plastic layer can conform to the device topography and sealed channels were obtained in spite of the existence of the sensors. However, the utilization of the polymeric material may give rise to compatibility problems if CMOS components are to be integrated. Furthermore, the packaging and handling of the comparatively compliant channel material may also require additional effort. In this work, a silicon-based MCE system integrated with feed-through driving electrodes has been designed and fabricated in an attempt to produce a robust microsystem free of most of the problems discussed.

\section{MCE device design and fabrication}

A conceptual design of an integrated MCE device is shown in figure 1. Glass and silicon wafers are used to utilize the advantages of both substrates. The transparent glass wafer allows external optical detection while the silicon wafer permits CMOS-compatible technology and provides

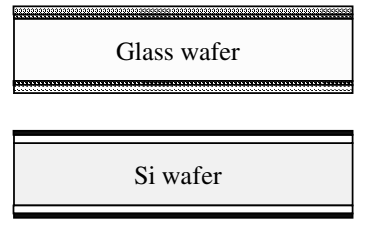

(a)

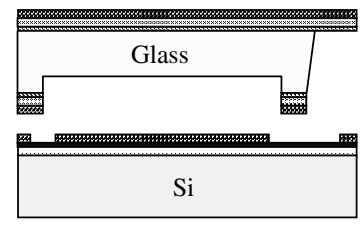

(c)

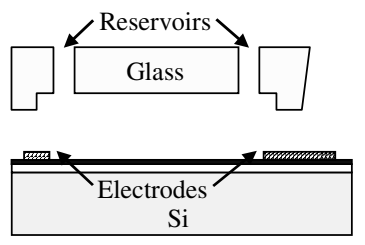

(e)

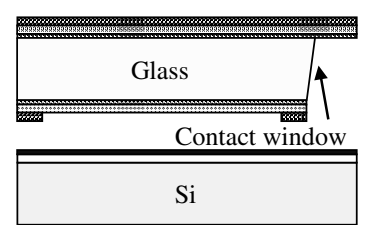

(b)

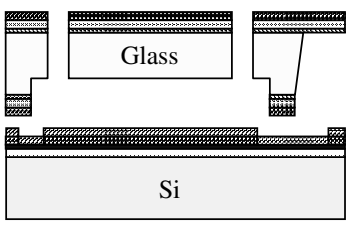

(d)

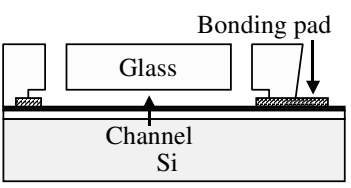

$(f)$
TiW $\quad \square$ Oxide a-Si $\mathrm{Pt} / \mathrm{TiW}$

Figure 2. Schematic cross-sections of the major fabrication steps.

faster heat dissipation. The glass is anodically bonded to a thick oxide layer on a silicon substrate utilizing an assisting amorphous silicon (a-Si) thin film. The successful anodic bonding of the thick silicon dioxide layer and glass resolves the early breakdown problem of anodically bonded siliconbased MCE systems. A thick layer is placed on the silicon wafer to insulate the semiconducting silicon substrate from the electrophoresis buffer. To provide the electric field needed for electrophoresis, driving electrodes are integrated into the device. All the metal interconnects are designed to terminate at the bonding pads placed on the silicon substrate, while wires are bonded to the pads through contact windows etched in the glass substrate. The integration of feed-through electrodes makes it possible to include additional sensors and circuits. Since no exotic materials are used, the conceptual system design is robust and allows further CMOS-compatible integration of on-chip devices.

\subsection{Device fabrication steps}

Schematic cross-sections of the major fabrication steps of the MCE device are shown in figure 2. The starting substrates were $100 \mathrm{~mm}$ p-type, (100) $\mathrm{Si}$ and $100 \mathrm{~mm}$ Corning 7740 glass wafers. A $1 \mu \mathrm{m}$ thick thermal oxide layer was first grown on the $\mathrm{Si}$ wafer for insulation. This was followed by low-pressure chemical vapour deposition (LPCVD) of a $\sim 10 \mathrm{~nm}$ thick a-Si thin film to significantly improve the yield and quality of glass to oxide anodic bonding (figure 2(a)). After removing the thin films from the back side of the Si wafer (figure 2(b)), lift-off photoresist was spun and patterned on the front side of the wafer with the electrode mask (figure 2(c)). Next, an $80 \mathrm{~nm}$ thick Pt layer with a $20 \mathrm{~nm}$ thick 


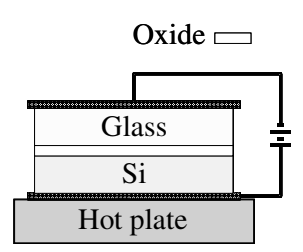

(a)

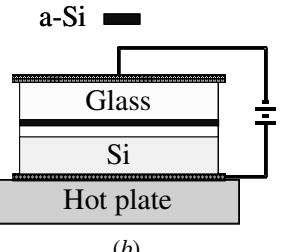

(b)
Figure 3. The set-up for glass-to-silicon anodic bonding with an interface of $(a)$ a thin oxide layer, and $(b)$ a thick oxide layer with a thin intermediate a-Si film.

TiW adhesion layer was sputtered on the wafer to form the electrodes and interconnects (figure $2(d)$ ), and then patterned by lifting off the photoresist mould (figure 2(e)).

In parallel, an etch mask consisting of a $400 \mathrm{~nm}$ thick $\mathrm{Au}$ layer with a $50 \mathrm{~nm}$ thick TiW adhesion layer was sputtered on both sides of the glass wafer and patterned with the interconnect mask (figure 2(a)). This was followed by spinning photoresist again on the patterned etch mask and, then, the channel and reservoir pattern was transferred to the photoresist but not to the $\mathrm{Au} / \mathrm{TiW}$ composite layer. After that, the glass wafer, together with the patterned etch mask and photoresist, was immersed in $49 \%$ hydrofluoric acid (HF) for $1.5 \mathrm{~h}$ to open contact windows for the wire bonds (figure $2(b)$ ). The photoresist was found to stick firmly to the etch mask even after the long HF etching. Therefore, it was subsequently used to transfer the channel and reservoir patterns to the etch mask. After pattern transfer to the Au/TiW composite layer, the glass wafer was etched again in $49 \%$ HF for 5 min to form $30 \mu \mathrm{m}$ deep channels and reservoirs (figure 2(c)). Drilling the access holes to the reservoirs (figure $2(d)$ ) and stripping all the remaining films (figure $2(e)$ ) completed the bulk micromachining of the glass wafer.

The two processed wafers were then bonded anodically under $900 \mathrm{~V}$ at $335^{\circ} \mathrm{C}$ for $1 \mathrm{~h}$ (figure $2(f)$ ). Although the $\mathrm{Pt}$ electrodes on the Si wafer incurred non-planar topography and a thick oxide layer existed on the Si wafer, hermetic and strong bonding was achieved and no leakage of fluid was observed in subsequent experiments. The bonded substrates were then cut and packaged on a printed circuit board (PCB) to complete the fabrication process.

\subsection{Glass-to-silicon anodic bonding with thick oxide at the interface}

Anodic bonding is a common technique in the fabrication of many microelectromechanical systems (MEMS) [14, 15]. A standard anodic bonding set-up is similar to that shown in figure 3(a), except for the absence of the oxide layer. At elevated temperatures, the $\mathrm{Na}^{+}$ions in the glass substrate become mobile enough such that they move towards the cathode of the applied high dc voltage. The relatively immobile $\mathrm{O}^{2-}$ ions are left behind at the silicon and glass interface. As the immobile oxygen anions continue to accumulate at that interface, a space-charge region is formed. In turn, an equivalent positive image charge is created on the silicon side at the interface resulting in a high electric field with magnitude up to $10^{6} \mathrm{~V} \mathrm{~cm}^{-1}$ at that location [16]. Under such high electric field, the oxygen anions at the space-charge

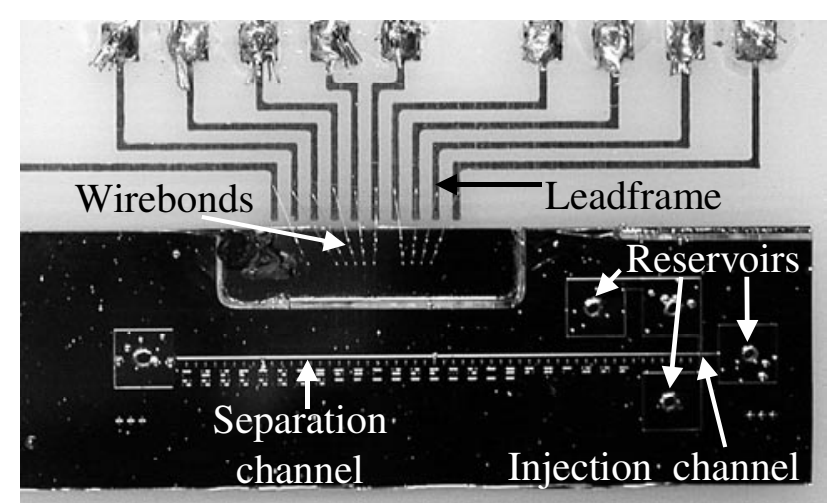

Figure 4. A photograph of a packaged integrated microsystem showing the microchannels, reservoirs and wire bonds.

region are believed to drift to the silicon side, oxidizing the silicon atoms. The thin oxide layer, thus formed, eventually binds the two substrates together.

Although anodic bonding can provide high bonding strength, its magnitude decreases with the thickness of an oxide layer on the silicon substrate. Hence, only a thin thermal oxide layer, up to a maximum of $3000 \AA$, is allowed to obtain strong bonding (figure $3(a)$ ). However, in MCE, high voltage is often used to achieve fast separation and good resolution of DNA fragments. Consequently, anodically bonded silicon-based microsystems have found little use in MCE due to the thin insulating oxide layer, which allows only low applied voltage. We have recently overcome this restriction [17]. With the newly developed amorphous-siliconassisted glass-to-silicon anodic bonding technology, silicon substrates with a thick insulating layer can be strongly bonded to glass substrates (figure 3(b)). The deposited thin a-Si layer is presumed to act as a substitute for the silicon atoms in the bulk silicon substrate during the bonding process and, hence, strong bonding strength can be achieved even if a thick oxide layer exists on the silicon substrate.

In the present work, this newly developed bonding technology was employed to construct the MCE devices. No leakage of liquid was observed during the pre-treatment and experimentation. This indicates that, despite the existence of a $1 \mu \mathrm{m}$ thick thermal oxide layer at the interface and the non-planar surface (100 nm thick integrated electrodes), hermetic and strong bonding has been successfully achieved. Subsequent experiments further showed that silicon substrates with a thicker insulating layer can also be bonded anodically to glass substrates using this technology.

\section{Experimental arrangements}

\subsection{The integrated MCE chip}

A fabricated MCE system is shown in figure 4. The microsystem adopts a five-reservoir, two-channel configuration. Among the five reservoirs, each about $3 \times$ $3 \mathrm{~mm}^{2}$ in area, four are integrated with driving platinum electrodes while the remaining reservoir is reserved for DNA loading. Access to the reservoirs is made possible through holes drilled in the glass substrate. The injection channel 


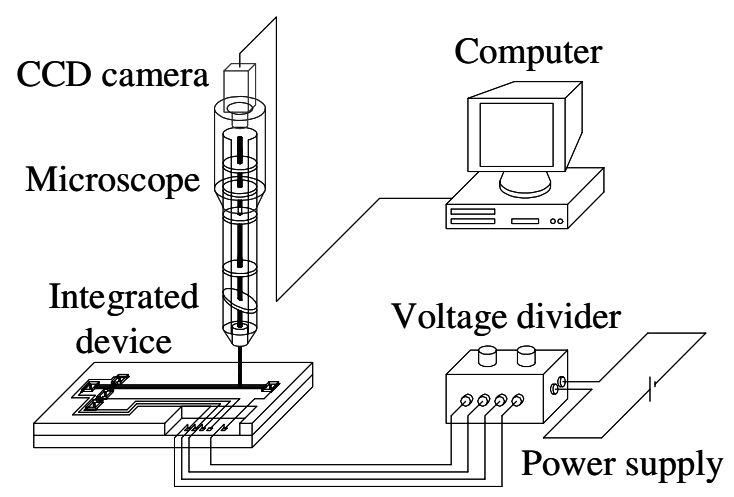

Figure 5. A schematic diagram of the experimental set-up for DNA electrophoresis.

delivers a certain amount of DNA sample from the loading reservoir to the intersection between the injection and the separation channels. Electrophoresis can then be performed along the separation channel upon the application of an electric field across the channel. The injection channel is relatively short, about $2 \mathrm{~mm}$, to prevent premature DNA separation. The separation channel is much longer, about $30 \mathrm{~mm}$, to provide good resolution between DNA fragments. The width of both channels is about $150 \mu \mathrm{m}$. A scale is placed next to the separation channel to indicate the distance migrated by the DNA fragments during the experiment. All the reservoirs, channels and scale markings are etched to a depth of $30 \mu \mathrm{m}$. A large contact window is etched through the glass substrate to keep the bonding pads exposed such that the MCE chip can be connected to a lead frame using wire bonds.

\subsection{Experimental set-up}

The experimental apparatus, schematically shown in figure 5, consists of two parts: (i) the microsystem chip with a main power supply and a voltage divider and (ii) the detection and image acquisition system. Agarose and hydroxyethylcellulose (HEC) dissolved in either $1.0 \times$ tris/acetate/EDTA (TAE) or $1.0 \times$ tris/borate/EDTA $(\mathrm{TBE})$ electrophoresis buffers were used as the sieving matrices in the experiments. The capillaries and reservoirs of the packaged MCE chip were loaded with the sieving matrix and then with fluorescein isothiocyanate (FITC) labelled 14-base single stranded oligonucleotide DNA or oxazole yellow dimer (YOYO-1) labelled Lambda DNA/EcoR I digest marker. The four integrated electrodes were connected to a voltage divider, which was powered by a main dc power supply. The voltage divider consisted of two circuits and relays arranged such that the voltages applied to the four reservoirs can be controlled with three adjustable potentiometers. In this arrangement, a pinched injection scheme was realized to obtain a well-defined injection plug [3], and instantaneous switching between injection and separation modes was possible during the experiments.

The detection and image acquisition system consisted of a Zeiss inverted microscope, and a CCD camera placed on a Gibraltar table that was connected to a computer with AXIS image acquisition and analysis program. The microscope was equipped with a halogen lamp and a mercury lamp, which allowed the visualization of the FITC (Ex/Em 494/518 nm)

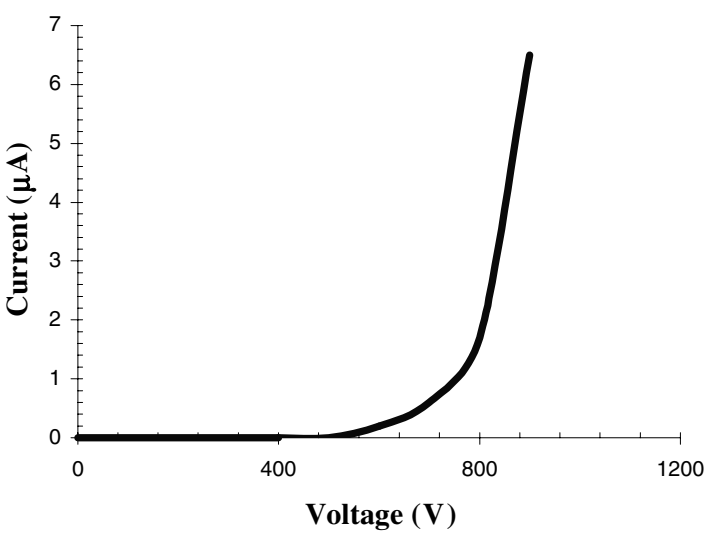

Figure 6. $I-V$ curve applied across a $1 \mu \mathrm{m}$ thick thermal oxide insulation layer.

and YOYO-1 (Ex/Em 491/509 nm) labelled DNA fragments during experiments. The images were acquired with the CCD camera and sent to the computer for in situ monitoring and image storage. Subsequent offline analysis on the captured images was then performed using the image acquisition and analysis program to investigate the DNA motion.

\section{Operational considerations}

\subsection{Voltage breakdown}

Voltage breakdown is a major concern in silicon-based MCE system. The electric field will pass through the sieving matrix only if that is the path with the lowest resistance in the system. The undoped a-Si layer cannot cause voltage breakdown due to two reasons: (i) the anodic bonding mechanism suggests that the thin a-Si layer should have been oxidized, and (ii) since the integrated electrodes were positioned very far apart (compared to the thickness of the oxide layer), the resistance of the a-Si is so high that voltage breakdown will most likely occur through the insulating oxide layer. A destructive voltage breakdown test of this oxide layer was performed by applying voltage across an integrated electrode and the silicon substrate, and the result is shown in figure 6 . Thermally grown silicon dioxide has a dielectric strength of around $5-10 \times 10^{6} \mathrm{~V} \mathrm{~cm}^{-1}$ depending on the conditions of the oxide growth [18]. The thickness of the oxide layer used in the current MCE devices was about $1 \mu \mathrm{m}$ which, theoretically, should sustain up to 500-1000 V before voltage breakdown occurs. The current-voltage $(I-V)$ curve measurements shown in figure 6 indicate that the voltage breakdown started at around $600 \mathrm{~V}$, which is within the calculated range. If a higher breakdown voltage is needed in the current system, a thicker insulating layer can be used. Subsequent bonding tests have demonstrated that, using the reported bonding technology, bonding with an alternating oxide/nitride composite layer of at least $4 \mu \mathrm{m}$ in thickness was possible ensuring higher breakdown strength.

\subsection{Bubble generation and transport}

Applying voltage to the integrated electrodes resulted in unavoidable electrolysis of the electrophoresis buffer, 


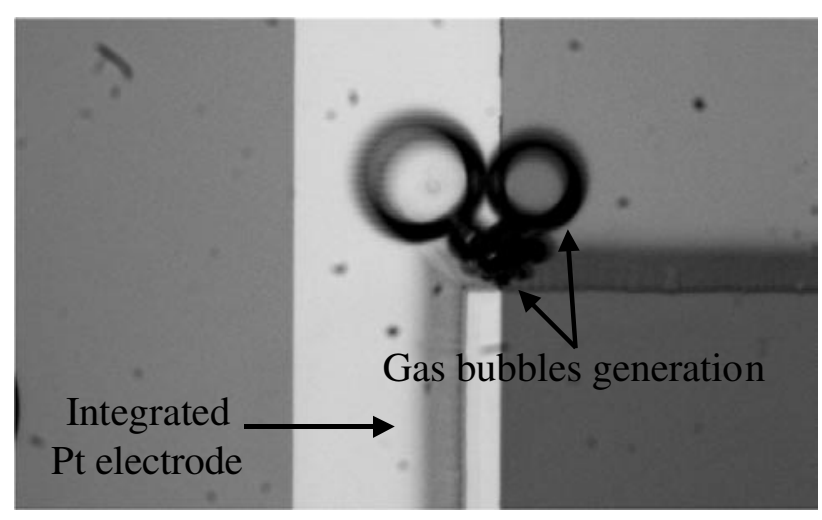

Figure 7. Gas bubble generation from an integrated electrode due to the electrolytic dissociation of the TBE buffer in the HEC sieving matrix.

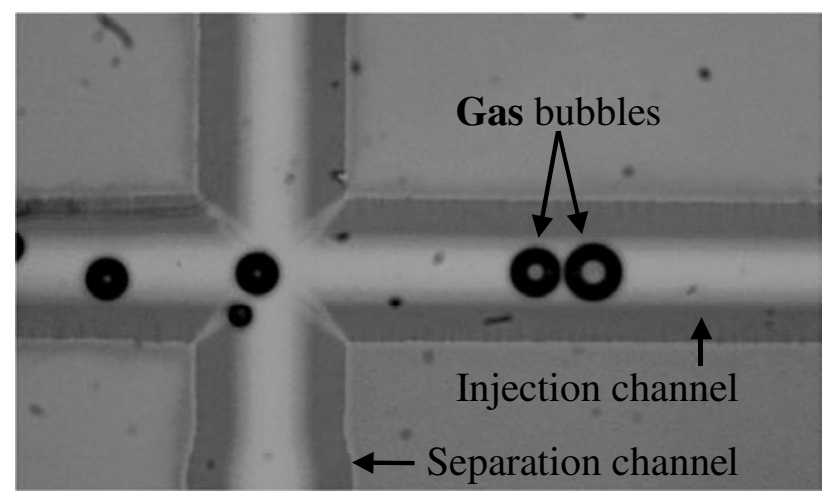

Figure 8. Small gas bubbles transported to the injection channel due to EOF prior to the polyacrylamide pre-treatment.

dissociating water into oxygen and hydrogen, as shown in figure 7. The gas bubbles thus formed have little effect on the experiment if they remain at the reservoirs or escape through the access holes. As long as the bubbles do not move into the channels or terminate the contact between the integrated electrodes and the sieving matrices, the electric field and hence the capillary electrophoresis can be maintained.

However, at times, small gas bubbles were transported to the injection channel, as shown in figure 8 . Bubbles in the channels resulted in a significant drop of the electrical current hindering the electrophoresis process. The material at the inner surface of both injection and separation channels is oxide, which normally carries negative charge. Therefore, upon the application of an electric field for electrophoresis, electro-osmotic flow (EOF) will also develop in the channels. This bulk fluid flow will transport into the channels the gas bubbles generated at the electrodes due to electrolysis. To solve this problem, a pre-treatment of the MCE devices was performed prior to the experiments. The capillary walls of the devices were coated following a modified Hjerten procedure $[19,20]$. The channels were filled with a $0.1 \mathrm{M}$ sodium hydroxide solution $\left(\mathrm{NaOH}_{(\mathrm{aq})}\right)$ for 10 min and then rinsed with deionized water. Commonly used for silica capillaries, this process renders the channel surfaces hydrophilic and hence facilitates subsequent loadings of the chemical pre-treatment

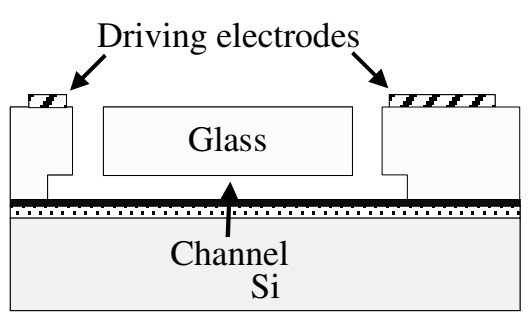

Figure 9. Schematic cross-section of the modified device design to eliminate gas bubble in the microsystem.

solutions. After drying the capillaries, a solution consisting of $15 \mu \mathrm{l}$ 3-methacryloxypropyltrimethoxysilane (bind-silane) in $0.5 \mathrm{ml}$ ethanol, adjusted to $\mathrm{pH} 3.6$, was injected into the channels. The solution was removed 10 min later and replaced with another fresh bind-silane solution for another $10 \mathrm{~min}$.

A freshly prepared $4 \%(\mathrm{w} / \mathrm{v})$ acrylamide solution containing $0.8 \mu \mathrm{l} N, N, N^{\prime}, N^{\prime}$-tetramethylenediamine (TEMED) and $2 \mathrm{mg}$ ammonium persulphate per $\mathrm{ml}$ was injected immediately after the removal of the second bind-silane. A vacuum pump was used to empty the channels once the viscosity of the solution had increased, indicating the formation of a linear polyacrylamide layer at the capillary walls. This pre-coating of the channel walls with a thin covalently bound layer of linear polyacrylamide suppressed the EOF because, at buffer $\mathrm{pH}$, the polyacrylamide carries very small surface charge. Consequently, the formation of the electric double layer, which is a precondition for EOF, is minimized.

Although it is difficult to completely eliminate the $\mathrm{pH}$ dependent EOF with the polyacrylamide pre-treatment, the gas bubbles did not migrate from the electrodes in pre-treated devices. This indicates the successful suppression of EOF by the thin linear polyacrylamide layer on the channel walls. The bubbles are negatively charged and their motion is due to both electro-osmosis and electrophoresis [21]. Without the pre-treatment, the EOF is strong enough to overcome the electrophoresis force on the bubbles resulting in bubble migration into the channels. Following the polymer coating, the EOF has been strongly suppressed but not completely eliminated. Thus, the weaker EOF can still balance the electrophoresis force such that the bubbles remain motionless. In addition to the EOF suppression, the polyacrylamide layer further facilitates the loading of the sieving matrix due to its hydrophilic nature. The coating was found to be stable, allowing multiple experiments without further treatment.

In order to completely eliminate the bubble presence inside the reservoirs and microchannels, a modified driving electrode design shown in figure 9 can be adopted. Rather than being placed at the bonding interface, the Pt electrodes can be re-located at the top surface of the glass substrate while other electrical components could be kept at the interface. In these microsystems, a large running buffer droplet is required engulfing the Pt driving electrode on top of each reservoir to maintain the electrical current path between the electrodes. The bubbles generated at the driving electrodes can escape directly to the surrounding due to buoyancy and, hence, no bubble can get into the reservoirs or channels. This approach 


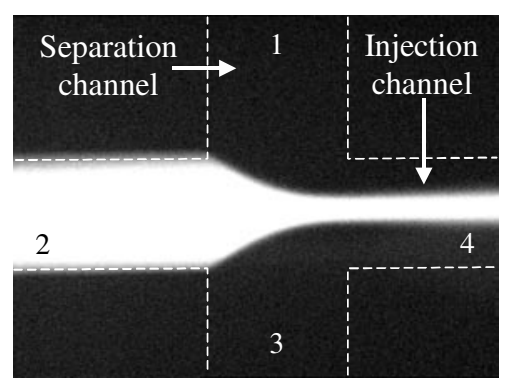

(a)

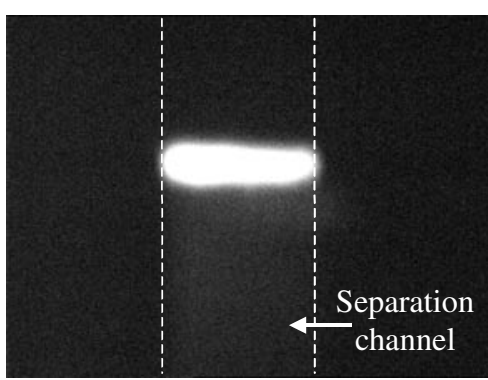

(b)

Figure 10. Pictures of DNA motion in the microsystem showing $(a)$ a pinched injection scheme, and $(b)$ a well-defined DNA plug in the separation channel.

completely prevents all the gas bubbles from entering the MCE system.

\section{Microsystem performance}

The pre-treated devices were loaded with HEC polymeric solution and FITC labelled 14-base DNA fragment. The DNA sample was injected into the intersection between the separation and injection channels by applying the injectionmode electric field across the four electrodes. In order to obtain a well-defined DNA plug in the separation channel, a pinched injection scheme shown in figure 10( $a$ ) was realized by properly adjusting the voltage level applied to each of the four electrodes using the voltage divider. In this injection mode, the voltage provided by the main dc power supply was $100 \mathrm{~V}$, corresponding to applied electric potentials of roughly $0,82,82$ and $100 \mathrm{~V}$ to the reservoirs connecting channels $1,2,3$ and 4 , denoted in figure $10(a)$, respectively. In this arrangement, the electric potential at the intersection of the injection and separation channels is estimated to be around $85.5 \mathrm{~V}$, resulting in field strengths of roughly $29,24,44$ and $97 \mathrm{~V} \mathrm{~cm}^{-1}$ in channels $1,2,3$ and 4, respectively. Switching the voltage divider from injection to separation mode resulted in the transportation of the DNA fragments from the intersection into the separation channel instantaneously. Under a proper adjustment of the electric potentials of about $100,78,0$ and $71 \mathrm{~V}$ (electric field strengths of 21, 274, 491 and $207 \mathrm{~V} \mathrm{~cm}^{-1}$, respectively) at the reservoirs connected to channels 1, 2, 3 and 4 respectively, for the same applied potential of $100 \mathrm{~V}$ from the main dc power supply, a welldefined DNA plug was formed in the separation channel as demonstrated in figure $10(b)$.

The DNA plug motion in the separation channel was captured by the CCD camera and recorded by a computer. Subsequent analysis was then performed using the image acquisition software. Figure 11 plots the light intensity distribution corresponding to a DNA plug travelling in the separation channel, used for determining the plug location. The velocity of the DNA plug can then be calculated from the distance travelled between two given frames and the elapsed time. The velocity dependence on the applied electric field is shown in figure 12 for 14-base DNA fragments using $2 \%$ agarose gel in TBE buffer as the sieving matrix. The linear curve suggests that the mobility, the slope of the curve, is

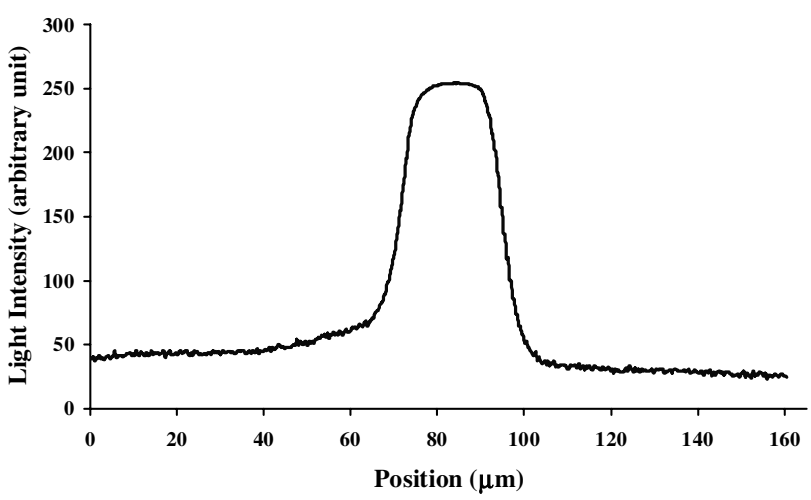

Figure 11. Light intensity distribution corresponding to the DNA plug shown in figure $10(b)$.

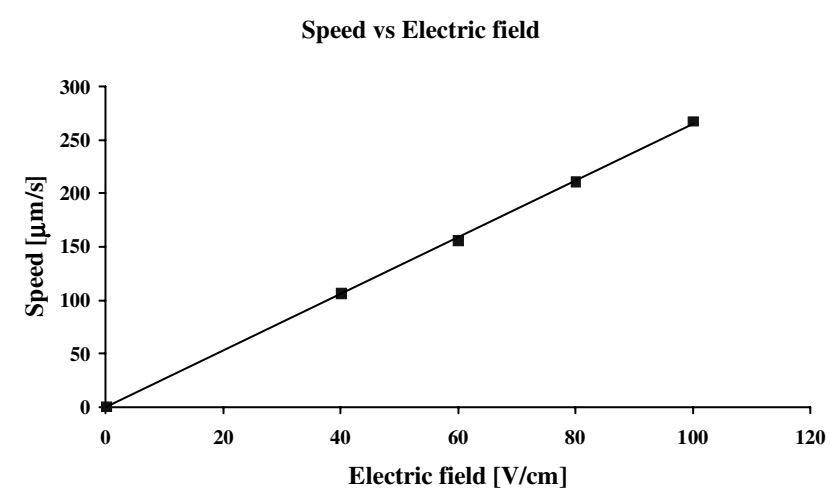

Figure 12. Speed dependence on the electric field of 14-base DNA fragments in $2 \%$ agarose.

independent of the electric field. This is consistent with results reported for small DNA fragments [22].

Finally, YOYO-1 labelled Lambda DNA/EcoR I digest marker was loaded into the microsystem to demonstrate DNA electrophoretic separation using $0.6 \%$ agarose gel in TAE buffer as the sieving matrix. The DNA plug was formed following the described pinched injection scheme. Upon switching the microsystem to separation mode, the welldefined plug entered the separation channel without premature separation under an applied electric field of $25 \mathrm{~V} \mathrm{~cm}^{-1}$ along the separation channel. The DNA plug clearly resolved 


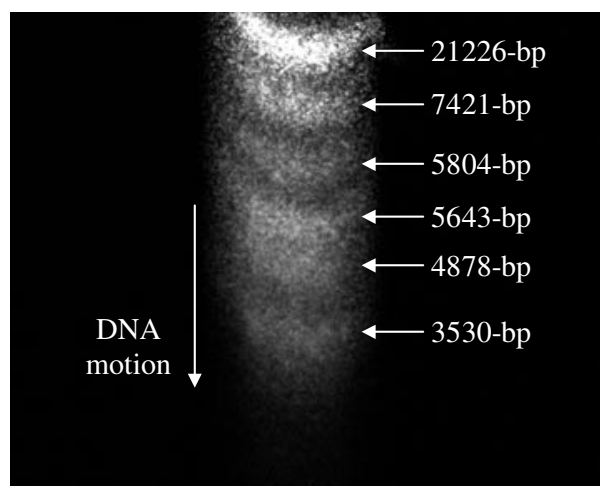

Figure 13. Electrophoretic separation of the YOYO-1 labelled Lambda DNA/EcoR I digest marker.

into six bands just $1.5 \mathrm{~min}$ after the switch to the separation mode, as shown in figure 13, matching the band pattern of EcoR I digested lambda DNA.

This satisfactory performance of the device in manipulating short DNA fragments and separating standard DNA markers suggests that silicon-based microsystems are practical for the fabrication of integrated MCE devices.

\section{Conclusions}

A novel silicon-based microsystem integrated with feedthrough driving electrodes has been successfully fabricated for the manipulation and separation of DNA samples. A new technology based on glass-to-silicon anodic bonding was utilized to fabricate the system. With this technology, a hermetic and strongly bonded integrated MCE system with a thick insulating layer has been realized. The successful bonding of glass to thick oxide on silicon resolves the early voltage breakdown problem of standard silicon-based MCE system. Moreover, the integration of feed-through electrodes excludes the requirement for external electrodes, which is very promising for further integration of on-chip DNA sensors and circuits. The breakdown voltage of the current microsystem, with a $1 \mu \mathrm{m}$ thick oxide layer, was found to be as high as $600 \mathrm{~V}$; a thicker insulating layer can be utilized if higher levels are required. Bubble generation, due to electrolysis, could be a major obstacle for MCE systems. Pre-coating the capillary walls with a thin linear polyacrylamide layer can practically neutralize the oxide negative surface charge and, thereby, suppress the EOF and avoid bubble transport into the channels. Furthermore, bubble presence in the microsystem could be completely eliminated by positioning the driving electrodes on the chip front side next to the reservoirs. Using a voltage divider, DNA manipulation has been demonstrated following the formation of a well-defined plug for DNA analysis. Electrophoretic separation of a standard DNA marker was also accomplished. The reported devices were found to be robust with no leakage of fluid during the experiments. Future integration of on-chip sensors and circuits will advance the device a further step to become a key part of a lab-on-a-chip microsystem.

\section{Acknowledgments}

This work is supported by the Hong Kong Research Grant Council through RGC grant HKUST6082/00E, the Industry Department of the Hong Kong SAR (AF/150/99) and the Hong Kong Jockey Club.

\section{References}

[1] Mastrangelo C H, Burns M A and Burke D T 1998 Microfabricated devices for genetic diagnostics Proc. IEEE 86 1769-87

[2] Harrison D J, Fluri K, Seiler K, Fan Z, Effenhauser C S and Manz A 1993 Micromachining a miniaturized capillary electrophoresis-based chemical analysis system on a chip Science 261 895-7

[3] Jacobson S C, Hergenroder R, Koutny L B, Warmack R J and Ramsey J M 1994 Effects of injection schemes and column geometry on the performance of microchip electrophoresis devices Anal. Chem. 66 1107-13

[4] Schmalzing D, Koutny L, Adourian A, Belgrader P, Matsudaira P and Ehrlich D 1997 DNA typing in thirty seconds with a microfabricated device Proc. Natl Acad. Sci. USA 94 10273-8

[5] Woolley A T, Lao K, Glazer A N and Mathies R A 1998 Capillary electrophoresis chips with integrated electrochemical detection Anal. Chem. 70 684-8

[6] Pumera M, Wang J, Opekar F, Jelinek I, Feldman J, Lowe H and Hardt S 2002 Contactless conductivity detector for microchip capillary electrophoresis Anal. Chem. 74 1968-71

[7] Laugere F, Berthold A, Lubking G W, Bastemeijer J, Guijt R M, Baltussen E, Sarro P M and Vellekoop M J 2001 Experimental verification of an improved method for conductivity detection in on-chip capillary electrophoresis systems Proc. Transducers'01 pp 1178-81

[8] Han J and Craighead H G 1999 Entropic trapping and sieving of long DNA molecules in a nanofluidic channel J. Vac. Sci. Technol. A 17 2142-7

[9] Spiering V L, van der Moolen J N, Burger G-J and van der Berg A 1997 Novel microstructures and technologies applied in chemical analysis techniques Proc. Transducers'97 pp 511-4

[10] Mogensen K B, Petersen N J, Hubner J and Kutter J P 2001 Monolithic integration of optical waveguides for absorbance detection in microfabricated electrophoresis devices Electrophoresis 22 3930-8

[11] Cozma A and Puers B 1995 Characterization of the electrostatic bonding of silicon and pyrex glass J. Micromech. Microeng. 5 98-102

[12] Webster J R, Burns M A, Burke D T and Mastrangelo C H 2000 Electrophoresis system with integrated on-chip fluorescence detection Proc. IEEE Micro Electro Mechanical Systems (MEMS) 2000 Conf. pp 306-10

[13] Selvaganapathy P, Burns M A, Burke D T and Mastrangelo C H 2001 Inline electrochemical detection for capillary electrophoresis Proc. IEEE Micro Electro Mechanical Systems (MEMS) 2001 Conf. pp 451-4

[14] Jiang L, Wong M and Zohar Y 2001 Forced convection boiling in a microchannel heat sink J. Microelectromech. Syst. 10 80-7

[15] Lee M, Wong Y Y, Wong M and Zohar Y 2002 Size and shape effects on two-phase flow instabilities in microchannels Proc. IEEE Micro Electro Mechanical Systems (MEMS) 2002 Conf. pp 28-31

[16] Wallis G 1970 Direct-current polarization during field-assisted glass-metal sealing J. Am. Ceram. Soc. 53 563-7

[17] Chan Y C, Lenigk R, Carles M, Sucher N J, Wong M and Zohar Y 2001 Glass-silicon bonding technology with 
feed-through electrodes for micro capillary electrophoresis Proc. Transducers'01 pp 1166-9

[18] Jaeger R C 1993 Introduction to Microelectronic Fabrication (Reading, MA: Addison-Wesley)

[19] Hjerten S 1985 High-performance electrophoresis elimination of electroendosmosis and solute adsorption J. Chromatogr. 347 191-8

[20] Hjerten S and Kiessling-Johansson M 1991 High-performance displacement electrophoresis in 0.025 - to $0.050-\mathrm{mm}$ capillaries coated with a polymer to suppress adsorption and electroendosmosis J. Chromatogr. $\mathbf{5 5 0}$ $811-22$

[21] Phianmongkhol A and Varley J $2003 \zeta$ potential measurement for air bubbles in protein solutions J. Colloid Interface Sci. $260332-8$

[22] Strutz K and Stellwagen N C 1998 Do DNA gel electrophoretic mobilities extrapolate to the free-solution mobility of DNA at zero gel concentration? Electrophoresis 19 635-42 